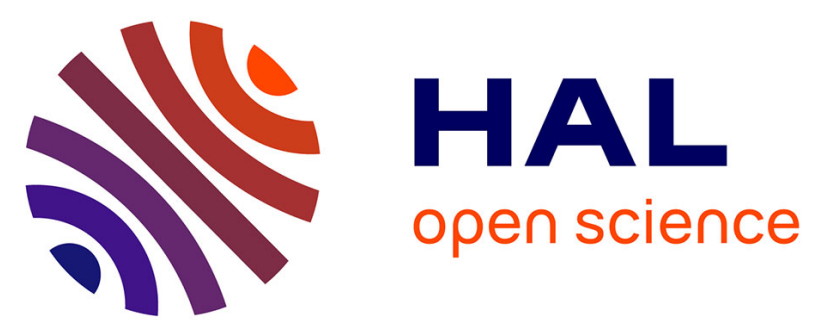

\title{
Comparison of three spatial differencing schemes in discrete ordinates method using three-dimensional unstructured meshes
}

\author{
D Joseph, Mouna El-Hafi, Richard A Fournier, B Cuenot
}

\section{- To cite this version:}

D Joseph, Mouna El-Hafi, Richard A Fournier, B Cuenot. Comparison of three spatial differencing schemes in discrete ordinates method using three-dimensional unstructured meshes. International Journal of Thermal Sciences, 2005, 44 (9), pp.851-864. 10.1016/j.ijthermalsci.2005.02.010 . hal01712158

\section{HAL Id: hal-01712158 \\ https://hal.science/hal-01712158}

Submitted on 8 Nov 2018

HAL is a multi-disciplinary open access archive for the deposit and dissemination of scientific research documents, whether they are published or not. The documents may come from teaching and research institutions in France or abroad, or from public or private research centers.
L'archive ouverte pluridisciplinaire HAL, est destinée au dépôt et à la diffusion de documents scientifiques de niveau recherche, publiés ou non, émanant des établissements d'enseignement et de recherche français ou étrangers, des laboratoires publics ou privés. 


\title{
Comparison of three spatial differencing schemes in discrete ordinates method using three-dimensional unstructured meshes
}

\author{
David Joseph ${ }^{\text {a }}$, Mouna El Hafi ${ }^{\mathrm{a}, *}$, Richard Fournier ${ }^{\mathrm{b}}$, Bénédicte Cuenot ${ }^{\mathrm{c}}$ \\ a Ecole des Mines d'Albi Carmaux, Campus Jarlard, route de Teillet, Albi, France \\ ${ }^{\mathrm{b}}$ Laboratoire Energétique, Université Paul Sabatier, 31077 Toulouse, France \\ ${ }^{\mathrm{c}}$ CERFACS 42, Av. Gaspard Coriolis, Toulouse, France
}

\begin{abstract}
A radiative heat transfer code based on the discrete ordinates method applied to unstructured grids has been developed to be coupled with a finite volume CFD code for combustion applications. The constraints are that: (1) Accurate coupling with a finite volume CFD code requires that the output is the integrated radiative source term within each mesh; (2) The resulting computation times must remain acceptable within the combustion requirements (of the order of an hour for realistic industrial geometries); (3) the line spectra of combustion gases must be accurately represented across the whole infrared range. Here, gaseous line spectra properties are represented with the SNB-ck model using narrow bands parallelization. The radiative transfer equation is discretized with a finite volume approach and three schemes are tested ("exponential", "step" and "diamond mean flux") in terms of accuracy and computational requirement. They are first tested for academic gray cases, solutions being compared to reference solutions provided by the Ray Tracing Method and the Monte Carlo Method. The behavior of the three schemes is also discussed for a spherical geometry, using an analytical solution in order to perform a parametric study of the absorption optical thickness influence in a wide range typical of spectral line gaseous radiation. Final tests involving a complete water vapor spectrum are performed in order to test the effects of preceding conclusions in terms of expected accuracies for combustion applications.
\end{abstract}

Keywords: Radiative transfer; Infrared line spectra; Discrete ordinates; Unstructured grids; Three-dimensional

\section{Introduction}

In computational fluid dynamics (CFD), the coupling between radiative heat transfer and combustion is based on the resolution of the energy equation. The heat source term due to radiation is evaluated by taking into account the temperature and radiating species concentration profiles, which are obtained from the solution of the aerothermochemistry equations. Among all the numerical methods developed to calculate the radiative heat transfer, the finite volume method (FVM) and the discrete ordinates method (DOM) offer good

\footnotetext{
* Corresponding author.

E-mail addresses: joseph@enstimac.fr (D. Joseph), elhafi@enstimac.fr (M. El Hafi), rfo@sphinx.ups-tlse.fr (R. Fournier), cuenot@cerfacs.fr (B. Cuenot).
}

compromises between accuracy and computational requirements. These approaches have been widely used to solve radiative transfer problems in structured three-dimensional geometries using Cartesian or cylindrical coordinates. In particular, the DOM, described by Chandrasekhar in 1950 [1], has been deeply studied by Lathrop and Carlson in 6070's [2] and by Truelove, Fiveland and Jamaluddin in the 80's [3-7]. Significant improvements have been achieved in the last decade aiming at the reduction of the ray effects and false scattering, more accurate quadratures and the extension to complex geometries. Nevertheless, the coupling between radiative transfer and other physical phenomena, such as combustion and fluid flow at high temperatures, requires the solution of the radiative transfer equation using the same grid employed to solve the other governing equations. 


\section{Nomenclature}

\begin{tabular}{|c|c|c|c|}
\hline$A$ & surface area $\ldots \ldots \ldots \ldots \ldots \ldots \ldots \mathrm{m}^{2}$ & $w$ & weight associated to a discrete direction \\
\hline$A_{\Delta}$ & $\begin{array}{l}\text { surface area of cell orthogonally planned } \\
\text { following } \mathbf{s}_{j} \ldots \ldots \ldots \ldots \ldots \ldots \ldots \ldots \ldots \mathrm{m}^{2}\end{array}$ & \multicolumn{2}{|c|}{ Greek symbols } \\
\hline$D_{i j}$ & 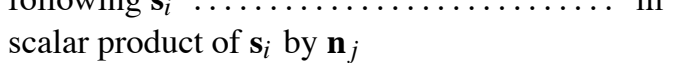 & $\alpha$ & weighting factor for mean flux scheme \\
\hline$G$ & incident radiation $\ldots \ldots \ldots \ldots$ & $\varepsilon$ & emissivity \\
\hline$I$ & radiation intensity $\ldots \ldots \ldots \ldots \ldots \ldots$ & $\kappa$ & absorption coefficient $\ldots \ldots \ldots \ldots \ldots \mathrm{m}^{-1}$ \\
\hline$N_{\text {dir }}$ & number of discrete directions & $\mu, \eta, \xi$ & director cosines of the discrete direction \\
\hline$N_{\text {face }}$ & number of faces of cells & $v$ & wave number $\ldots \ldots \ldots \ldots \ldots \ldots \ldots \mathrm{m}^{-1}$ \\
\hline$Q_{w}$ & net heat flux at the wall $\ldots \ldots \ldots \ldots W \cdot \mathrm{m}^{-2}$ & $\Sigma$ & surface area delimiting a volume $\ldots \ldots \ldots \mathrm{m}^{2}$ \\
\hline$R$ & radius $\ldots \ldots \ldots \ldots \ldots \ldots \ldots \ldots \ldots \ldots \ldots \ldots \ldots$ & & \\
\hline$S_{r}$ & radiative source term $\ldots \ldots \ldots \ldots \ldots W \cdot \mathrm{m}^{-3}$ & $S Z$ & solid angle $\ldots \ldots \ldots \ldots \ldots \ldots \ldots$ \\
\hline$T$ & temperature $\ldots \ldots \ldots \ldots \ldots \ldots \ldots \ldots \ldots$ & \multicolumn{2}{|c|}{ Subscripts } \\
\hline$\underline{V}$ & volume $\ldots \ldots \ldots \ldots \ldots \ldots \ldots \ldots \ldots \mathrm{m}^{3}$ & A & beginning point of a pathway through a cell \\
\hline$\overline{I_{\text {in }}}$ & averaged intensity over entries $\ldots . . \mathrm{W} \cdot \mathrm{m}^{-2} \cdot \mathrm{sr}$ & $\mathrm{B}$ & ending point of a pathway through a cell \\
\hline$\overline{I_{\text {out }}}$ & averaged intensity over exit faces $\ldots \mathrm{W} \cdot \mathrm{m}^{-2} \cdot \mathrm{sr}$ & $b$ & blackbody \\
\hline $\begin{array}{l}\mathbf{Q}_{r} \\
\mathbf{n}\end{array}$ & $\begin{array}{l}\text { radiative heat flux vector } \ldots \ldots \ldots \ldots . \mathrm{W} \cdot \mathrm{m}^{-2} \\
\text { unit vector normal to the face }\end{array}$ & $i$ & $\begin{array}{l}\text { associated to } i \text { th discrete direction of the } \\
\text { angular quadrature }\end{array}$ \\
\hline $\mathbf{S}$ & discrete direction unit vector & $j$ & associated to $j$ th face of the cell \\
\hline$f_{v}$ & soot volumetric fraction & $k$ & associated to $k$ th entry of the cell \\
\hline$h$ & height $\ldots \ldots \ldots \ldots \ldots \ldots \ldots \ldots \ldots$ & $l$ & associated to $l$ th exit face of the cell \\
\hline$l_{\max }$ & maximum thickness of a cell $\ldots$. & $P$ & associated to the volume of the cell \\
\hline$s$ & coordinate along direction $\mathbf{s}$ & $w$ & wall \\
\hline$t$ & optical pathlength through a cell $\ldots \ldots \ldots \ldots$ m & $x, y, z$ & Cartesian coordinates \\
\hline
\end{tabular}

Unstructured grids are often used in CFD owing to their geometrical flexibility. In this way, a lot of work has been developed during the last decade to apply the DOM-FVM to non-orthogonal structured grids and unstructured grids in three-dimensional enclosures [8-11,15]. In particular, Sakami and co-workers proposed an accurate method for the spatial discretization by taking into account the exponential extinction [12-14], but it necessitates to perform a heavy preprocessing procedure. Much less sophisticated schemes are also commonly used. Liu et al. [15] have used the "step" scheme, equivalent of the "upwind" scheme in CFD and Ströhle et al. [17] have proposed the mean flux interpolation scheme. Another type of spatial discretization was also recently introduced, namely the Discrete Ordinates Interpolation Method (DOIM), which does not rely upon an integration of the radiative transfer equation over the control volumes, but rather on the integration of the equation along a line of sight. This method has been introduced by Seo et al. in 1998 [18] and extended to unstructured grids by Cha et al. in 2000 [19]. Lastly, Koo et al. [20] have compared three methods applied to two-dimensional curved geometries: the DOIM, Sakami's approach and the discrete ordinates method in orthogonal curvilinear coordinates [21].

In our study, a computer code using unstructured meshes and based on the modeling of radiative transfer using the DOM has been developed aiming at a future coupling with a combustion finite volume code available at the CERFACS ${ }^{1}$ in France. The code has been specially written for unstructured grids using tetrahedrical cells, by trying to avoid complex adaptations that are highly time consuming. The constraints associated to combustion applications where the following:

(1) Accurate coupling with a finite volume CFD code requires that the output is the integrated radiative source within each mesh;

(2) The resulting computation time must remain acceptable for realistic industrial geometries in combustion;

(3) The line spectra of combustion gases must be accurately represented across the whole infrared range.

Then, to treat general combustion situations, even if the main part of this paper is devoted to gray media, all these constraints lead to the following choices:

(1) Gaseous line spectra properties are represented with a Statistical Narrow Band correlated- $k$ model and parallelization is used to simultaneously compute the radiative contribution of each narrow band;

\footnotetext{
${ }^{1}$ Centre Européen de Recherche et de Formation Avancée en Calcul Scientifique.
} 
(2) For each correlated- $k$ computation the radiative transfer equation is discretized with a finite volume approach (in order to avoid additional computational efforts when coupling with the finite volume combustion code) and three spatial differencing schemes are considered: "exponential" [13], "step" [15] and "diamond mean flux" [17].

The performances of the different spatial discretization schemes have already been widely investigated in cases of simple geometries mapped using Cartesian coordinates, but there is a lack of information regarding the performance of these schemes with unstructured grids. In the present study, after some theoretical derivations (Section 2), we analyze the behavior of the three retained schemes on academic configurations (Section 3). Results are compared with accurate solutions that are analytical or produced by the Ray Tracing Method. In this analysis convergence difficulties are identified in the limit of strong absorption optical thicknesses.

These difficulties are further analyzed using a parametric study to cover the wide range of absorption optical thicknesses typical of spectral line gaseous radiation. A particular attention is given to the standard finite volume approximation and its convergence difficulties at high absorption optical thicknesses, independently of the spatial differencing scheme. We then consider configurations with real gaseous line spectra for the whole infrared range in order to explore the effect of such convergence difficulties for combustion applications. This last case, with a complete $\mathrm{H}_{2} \mathrm{O}$ line spectrum, allows to draw first conclusions and to comment on the validity range of three spatial differencing schemes for combustion applications.

\section{The radiative transfer equation (RTE)}

\subsection{Mathematical formulation}

Considering an absorbing-emitting and non-scattering gray medium, the variation of the radiative intensity along a line of sight can be written as:

$\frac{\mathrm{d} I(\mathbf{s})}{\mathrm{d} s}=\kappa I_{b}-\kappa I(\mathbf{s})$

where $I(\mathbf{s})$ is the radiative intensity, $I_{b}$ the radiative intensity of the blackbody, and $\kappa$ the absorption coefficient. Boundary conditions for diffuse surfaces are taken from the relation giving the intensity leaving the wall $I_{w}$ as a function of the blackbody intensity of the wall $I_{b, w}$ and of the incident radiative intensity:

$I_{w}(\mathbf{s})=\varepsilon_{w} I_{b, w}+\frac{1-\varepsilon_{w}}{\pi} \int_{\mathbf{n} . \mathbf{s}^{\prime}<0} I_{w}\left(\mathbf{s}^{\prime}\right)\left|\mathbf{n} . \mathbf{s}^{\prime}\right| \mathrm{d} \Omega^{\prime}$

where $\varepsilon_{w}$ is the wall emissivity, $\mathbf{n}$ the unit vector normal to the wall and $\mathbf{s}^{\prime}$ the direction of propagation of the incident radiation confined within a solid angle $\mathrm{d} \Omega^{\prime}$.

\subsection{Angular discretization}

The DOM is based on the discretization of the radiative transfer equation (RTE) according to a chosen number $N_{\text {dir }}$ of discrete directions, $\mathbf{s}_{i}\left(\mu_{i}, \eta_{i}, \xi_{i}\right)$ associated with their respective weights $w_{i}$, contained in the solid angle $4 \pi$. In this way, different angular discretizations can be used. A recent study carried out by Koch and Becker [22] compares the efficiency of several types of angular quadratures widely outside the most common ones. In this study, we choose the $S_{N}$ quadrature, which is one of the most popular and, in order to estimate the role of ray effect, some tests are performed with the $L C_{11}$ quadrature as recommended by Koch and Becker.

\subsection{Spatial discretization for unstructured grids}

The RTE is solved for every discrete direction $\mathbf{s}_{i}$ using a finite volume approach. The integration of the RTE over the volume $V$ of an element limited by a surface $\Sigma$, and the application of the divergence theorem yield:

$\int_{\Sigma} I\left(\mathbf{s}_{i}\right) \cdot \mathbf{s}_{i} \cdot \mathbf{n} \mathrm{d} \Sigma=\int_{V}\left(\kappa I_{b}-\kappa I\left(\mathbf{s}_{i}\right)\right) \mathrm{d} V$

We assume that $I_{b}$ and $I\left(\mathbf{s}_{i}\right)$ are constant over the volume $V$ and equal to their respective averaged value: $I_{b, P}$ and $I_{P}\left(\mathbf{s}_{i}\right)$. We also assume that the intensities at the faces are constant over each face and $I_{j}$ denotes the intensity at the $j$ th face. For unstructured grids, the domain is discretized in tetrahedra and every other hybrid grid can be under-meshed by tetrahedra. Then, for tetrahedra, Eq. (3) is discretized as:

$\sum_{j=1}^{N_{\text {face }}=4} I_{j}\left(\mathbf{s}_{i}\right) \cdot\left(\mathbf{s}_{i} \cdot \mathbf{n}_{j}\right) A_{j}=\kappa V\left(I_{b, P}-I_{P}\left(\mathbf{s}_{i}\right)\right)$

where $\mathbf{n}_{j}$ is the exit unit vector normal to the surface $j$.

The scalar product of the $i$ th discrete direction vector with the normal vector of the $j$ th face of the considered tetrahedron is defined by $D_{i j}$ :

$D_{i j}=\mathbf{s}_{i} \cdot \mathbf{n}_{j}=\mu_{i} n_{x j}+\eta_{i} n_{y j}+\xi_{i} n_{z j}$

The discretization of the boundary condition (Eq. (2)) is straightforward:

$I_{w}=\varepsilon_{w} I_{b, w}+\frac{1-\varepsilon_{w}}{\pi} \sum_{\mathbf{n} . \mathbf{s}_{i}<0} w_{i} I\left(\mathbf{s}_{i}\right)\left|\mathbf{n} . \mathbf{s}_{i}\right|$

For each cell, the incident radiation $G$ is evaluated as follows:

$G=\int_{4 \pi} I\left(\mathbf{s}_{i}\right) \mathrm{d} \Omega \simeq \sum_{i=1}^{N_{\mathrm{dir}}} w_{i} I_{P}\left(\mathbf{s}_{i}\right)$

For a gray medium, the divergence of the radiative heat flux $S_{r}$ is given by

$S_{r}=\nabla \cdot \mathbf{Q}_{r}=\kappa\left(4 \pi I_{b}-G\right)$

and the radiative net heat flux at the wall is calculated by 
$Q_{w} \simeq \varepsilon\left(\pi I_{b, w}-\sum_{\mathbf{n} . \mathbf{s}_{i}<0} w_{i} I\left(\mathbf{s}_{i}\right)\left|\mathbf{n} . \mathbf{s}_{i}\right|\right)$

For the three different spatial differencing schemes used, calculations of $I\left(\mathbf{s}_{i}\right)$ are detailed in the next sections.

\subsubsection{The exponential scheme}

Sakami et al. proposed in 1998 [13,14] a sophisticated spatial scheme for three-dimensional cases that takes into account the exponential extinction along the optical pathway into the cell. Considering an optical path $t=s_{\mathrm{B}}-s_{\mathrm{A}}$, where $s_{\mathrm{A}}$ and $s_{\mathrm{B}}$ are respectively the positions at the entry and at the exit of the cell, the solution of the RTE (Eq. (1)) can be written as

$I\left(\mathbf{s}, s_{\mathrm{B}}\right)=I\left(\mathbf{s}, s_{\mathrm{A}}\right) e^{-\kappa t}+\kappa \int_{s_{\mathrm{A}}}^{s_{\mathrm{B}}} I_{b} e^{-\kappa\left(s_{\mathrm{B}}-s\right)} \mathrm{d} s$

The integration over the cell pathlength leads to

$I\left(\mathbf{s}, s_{\mathrm{B}}\right)=I\left(\mathbf{s}, s_{\mathrm{A}}\right) e^{-\kappa t}+\left(1-e^{-\kappa t}\right) I_{b}$

For a direction $\mathbf{s}_{i}$ and a final position $s_{\mathrm{B}}$ at the exit face, the position $s_{\mathrm{A}}$ at the entry and the associated pathlength $t$ are fixed. The intensity $I_{l}$ going through an exit face $l$ is defined as the averaged intensity over the $l$ th face:

$I_{l}=\frac{1}{A_{l}} \iint_{A_{l}}\left[I\left(\mathbf{s}_{i}, s_{\mathrm{A}}\right) e^{-\kappa t}+I_{b}\left(1-e^{-\kappa t}\right)\right] \mathrm{d} A_{l}$

It has been shown in [13] that, knowing $\tau_{i}=\kappa l_{\max , i}$, where $l_{\max , i}$ stands for the maximum thickness of the cell for the direction $\mathbf{s}_{i}$, for the three different types of events presented in Fig. 1, one can calculate intensity $I_{l}$ at an exit face as follows:

$I_{l}=\left(\sum_{\substack{k \\ D_{i k}<0}} \frac{A_{l k}}{A_{l}} I_{k}\right) \chi_{i}+I_{b}\left(1-\chi_{i}\right)$

where $A_{l}$ is the exit surface area, $A_{l k}$ stands for the part of surface area of the downstream face $l$ obtained by projection of the upstream face $k$ on the face $l$ according to $\mathbf{s}_{i}$ and $\chi_{i}$ is a typical coefficient that accounts for the exponential extinction through the cell:

$\chi_{i}=\frac{1}{A_{l}} \int_{A_{l}} e^{-\kappa t} \mathrm{~d} A_{l}=\frac{2}{\tau_{i}}\left(1-\frac{1-e^{-\tau_{i}}}{\tau_{i}}\right)$

The difficulty lies in the calculation of the geometric maximum thickness $l_{\max , i}$ for all the cases presented in the Fig. 1 . $l_{\max , i}$ can be determined if we know the coordinates of the points A and B. By referring to Eq. (4), $I_{P}$ is directly deduced:

$I_{P}=I_{b, P}-\frac{1}{\kappa V} \sum_{j=1}^{N_{\mathrm{face}}=4} D_{i j} A_{j} I_{j}$

Whereas this scheme avoids negative values for all $I_{j}$ at cell faces, it does not guaranty that $I_{P}$ is positive at the center of each cell, in particular when $\kappa$ becomes very small.
First type of event : 3 entries

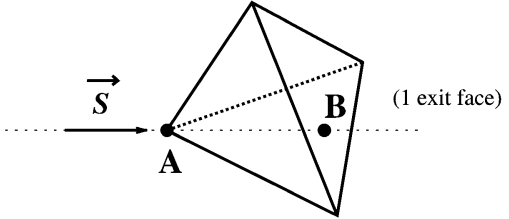

Second type of event : 2 entries

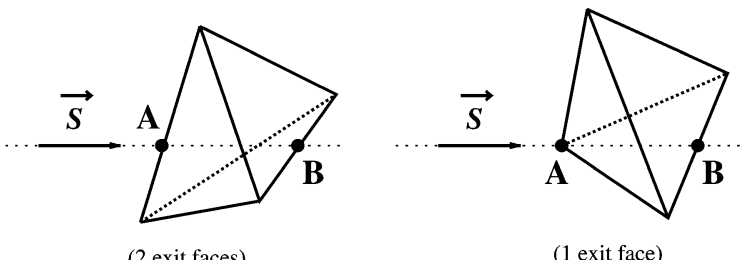

Third type of event : 1 entry

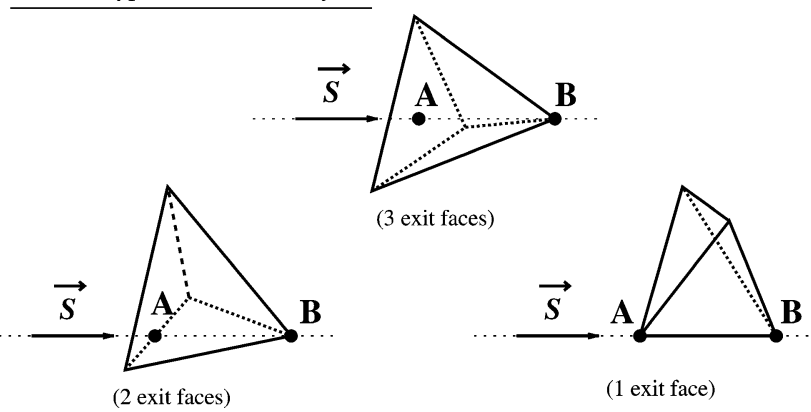

Fig. 1. Six ways to cross a tetrahedrical cell from A to B according to the number of entries and exit faces.

\subsubsection{The step scheme}

Much less sophisticated schemes are also available that are worth a detailed attention in our specific context. Considering our constraints in terms of computational times, the use of the exponential scheme will indeed be only justified if it insures significantly higher accuracy levels for combustion applications. In 2000 [15], J. Liu used the "step" scheme, which corresponds to the "Upwind" scheme that is commonly used in CFD. This scheme had already been proposed by Chai et al., in 1995 [9], in order to solve the RTE by the FVM for irregular geometries using curvilinear coordinates. In previous studies, this scheme has often been applied to Cartesian structured grids, in order to avoid negative values that could occur with schemes such as the "diamond" one [16]. Omitting the scattering phenomenon, the intensity $I_{P}$ is evaluated at the center of the cell by applying:

$$
I_{P}=\left[\kappa V I_{b}-\sum_{\substack{j=1 \\ D_{i j}<0}}^{N_{\text {face }}} D_{i j} A_{j} I_{j}\right] /\left[\kappa V+\sum_{\substack{j=1 \\ D_{i j}>0}}^{N_{\text {face }}} D_{i j} A_{j}\right]
$$


where $N_{\text {face }}$ is the number of faces for the cell (equal to 4 in our case). The downstream surface intensities are set equal to the intensity $I_{P}$.

\subsubsection{The mean flux scheme}

In a similar way, Ströhle et al. [17] proposed a simple spatial differencing scheme based on the mean flux scheme that can be very useful in the case of unstructured grids. This scheme relies on the following formulation:

$I_{P}=\alpha \overline{I_{\text {out }}}+(1-\alpha) \overline{I_{\text {in }}}$

where $\overline{I_{\text {in }}}$ and $\overline{I_{\text {out }}}$ are the cell face averaged intensities entering (white cell faces in Fig. 2) and leaving (gray cell face in Fig. 2) the control volume, respectively:

$\overline{I_{\text {in }}}=\left[\sum_{\substack{j \\ D_{i j}<0}} D_{i j} A_{j} I_{j}\right] /\left[\sum_{\substack{j \\ D_{i j}<0}} D_{i j} A_{j}\right]$

and

$\overline{I_{\mathrm{out}}}=\left[\sum_{\substack{j \\ D_{i j}>0}} D_{i j} A_{j} I_{j}\right] /\left[\sum_{\substack{j \\ D_{i j}>0}} D_{i j} A_{j}\right]$

Substituting $\overline{I_{\text {in }}}$ from Eq. (17) into Eq. (4) yields, after some algebra:

$$
\begin{gathered}
I_{P}=\left[\alpha V \kappa I_{b}-\Theta \sum_{\substack{j \\
D_{i j}<0}} D_{i j} A_{j} I_{j}\right] \\
/\left[\alpha \kappa V+\sum_{\substack{j \\
D_{i j}>0}} D_{i j} A_{j}\right]
\end{gathered}
$$

with

$\Theta=\alpha-(1-\alpha)\left[\sum_{\substack{j \\ D_{i j}>0}} D_{i j} A_{j}\right] /\left[\sum_{\substack{j \\ D_{i j}<0}} D_{i j} A_{j}\right]$

As shown in Fig. 2, if we consider that the scalar product $D_{i j}$ is the transformation term of the projection of the surface

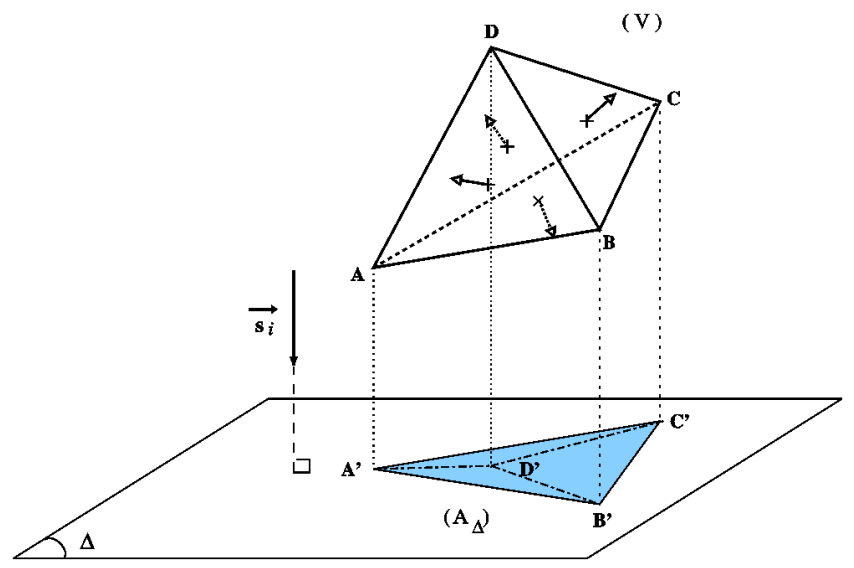

Fig. 2. Geometrical transformation linked to the term $D_{i j}$.
$A_{j}$, following the vector $\mathbf{s}_{i}$, on the plane $\Delta$ orthogonal to this last vector, we can write:

$$
\sum_{\substack{j \\ D_{i j}>0}} D_{i j} A_{j}=-\sum_{\substack{j \\ D_{i j}<0}} D_{i j} A_{j}=A_{\Delta}
$$

Then for all $\alpha$ in ]0, 1], we have $\Theta=1$ and finally, Eq. (20) takes the following form:

$$
I_{P}=\left[\alpha V \kappa I_{b}-\sum_{\substack{j \\ D_{i j}<0}} D_{i j} A_{j} I_{j}\right] /\left[\alpha \kappa V+\sum_{\substack{j \\ D_{i j}>0}} D_{i j} A_{j}\right]
$$

The case $\alpha=1$ corresponds to the Step scheme (Eq. (16)) used by Liu et al. [15]. The case $\alpha=0.5$ will be called diamond mean flux scheme (DMFS). It can be compared to the diamond scheme used in structured grids but it had been shown that the DMFS used in structured grid is different from the standard diamond scheme because of the mean flux approximation [17]. It is formally more accurate than the step scheme. After calculation of $I_{P}$ from Eq. (20), the radiation intensities at cell faces such that $D_{i j}>0$ are set equal to $\overline{I_{\text {out }}}$, obtained from Eq. (17). It can occur that this last term calculated is negative. $\overline{I_{\text {out }}}$ negative is equivalent to the following conditions:

$I_{b, P}<\left((1-\alpha)-\frac{A_{\Delta}}{\kappa V}\right) \cdot \overline{I_{\text {in }}}$

\subsection{Sweeping order optimization}

For a given discrete direction, each plane face of each cell is either an upstream face or a downstream face (a face parallel to the considered discrete direction plays no role). The control volumes should be treated following a sweeping order such as the radiation intensities at upstream cell faces are known. This order depends on the discrete direction under consideration. An algorithm for the optimization of the sweeping order has been implemented in the present work. To order the cells, we have to define first the direction $\mathbf{s}_{i}$. To proceed to the ordering, the grid is swept a first time in an arbitrary order with checking, for each cell, if the number of upstream faces where the intensity is known is the same as the number of upstream faces. If this condition is satisfied for one cell, the number of this cell is stored in a list for the direction $\mathbf{s}_{i}$. Then, the intensity of the downstream cell faces can be known, as the upstream cell faces of the next cells which are updated. We should notice that this sweeping order stored for each discrete direction, only depends on the chosen grid and the angular quadrature. That means that the use of different physical parameters do not change the established list of the sweeping order. A sweeping order optimization avoids too many iterations not only for the cases with scattering media and/or reflective walls geometries, but also avoids iteration in the case of black walled enclosures without scattering. 


\section{Results and discussion}

To compare the efficiency of the spatial differencing schemes for different types of enclosures containing a participating and homogeneous medium, several test cases have been carried out.

It should be pointed out that for output purposes an interpolation is performed on the results obtained with the unstructured code, in order to have the values for a given axis or a given point. This 3D-interpolation can generate inaccuracies, especially for the points taken near the walls or when the grid is coarse.

\subsection{Application to a black walled cylindrical enclosure}

This test concerns the cylindrical geometry to illustrate the influence of the angular quadrature on the radiative heat flux at the walls.

A cylinder ( $h=3 \mathrm{~m}$ and $R=0.5 \mathrm{~m}$ ) containing a gray isothermal medium at $T=1200 \mathrm{~K}$ is considered. The walls are black and at $T_{w}=300 \mathrm{~K}$. The radiative heat source $S_{r}$ along the axis of the cylinder and the radiative net heat flux $Q_{w}$ at the side wall are obtained with the unstructured code using the three different spatial discretization schemes described previously and the $S_{8}$ quadrature is employed. Two unstructured grids are used in this test case (see Fig. 3). A coarse one comprises 4000 tetrahedra and a finer one 210000 tetrahedra. A third comparison is made on the same fine grid using a more accurate angular discretization $\left(S_{12}\right)$. Results are compared to those obtained with the ray tracing method using 320000 rays. In the case of a homogeneous isothermal medium, the results of the ray tracing method are independent of the grid.

Two different values for the absorption coefficient of the medium have been chosen to represent weak $\left(\kappa=0.1 \mathrm{~m}^{-1}\right)$ and strong $\left(\kappa=10.0 \mathrm{~m}^{-1}\right)$ optical thicknesses. It should be noticed that the optical thickness of the cells also depends on the grid refinement. In the case of optically thin media (see Fig. 4), the peak of the net heat flux at the wall predicted by the DOM is about $10 \%$ lower than the ray tracing solu-

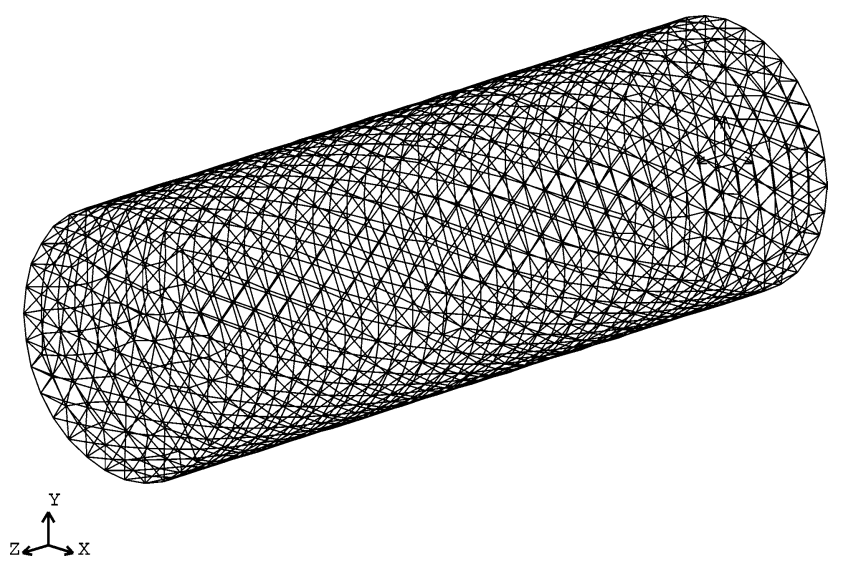

Fig. 3. Cylindrical enclosure grid. tion. This is due to the fact that the distribution of optical thicknesses in the medium is function of the angular direction and consequently needs a good angular representation (what can be linked to the well known ray effect). Increasing the order of the angular quadrature (Fig. 4(c)) improves the accuracy of the results. However, such an order of accuracy is compatible with today's expectations in combustion problems. For stronger optical thicknesses (see Fig. 5), the ray effect is softened. The aforesaid distribution of optical thicknesses is less sensitive to angular variation. For optically thick media, the increase of the absorption coefficient of the medium yields also an increase of the wall heat flux. The walls receive the radiation mainly from the closest cells of the medium, so there is no need to refine the grid and the angular discretization. In the case of optically thin media (see Fig. 4), the radiative heat source term solutions are in good agreement. Refining the grid gives better results. Increasing the absorption coefficient of the medium leads to decrease the accuracy obtained for the radiative source term solutions (see Fig. 5(a)). The relative difference between the DOM and the ray tracing solutions is negligible in the vicinity of the walls and becomes significant far from the walls. Most of the energy emitted by the medium is absorbed within a short distance. That leads to a strong exponential extinction that none of the three spatial differencing schemes succeeded in representing if the grids are not sufficiently fine (Figs. 4(a) and 5(a)). So, in the next section, a particular attention will be paid to the accuracy on the radiative source term estimation in a simpler configuration. The step scheme gives better results in optically thin case and the DMFS is the most accurate for very absorbing medium. The angular refinement does not really govern the accuracy of the source term along the centerline. We have noticed that the grid refinement influence is stronger than the angular one when we compare the three spatial differencing schemes.

Maximal, mean and minimal relative errors for the source term given in Tables 1 and 2 are computed for the three different spatial differencing schemes as follows

$E=\left|\frac{S_{r, \mathrm{DOM}}-S_{r, \mathrm{RT}}}{S_{r, \mathrm{RT}}}\right| \times 100$

and for the wall heat flux:

$E=\left|\frac{Q_{w, \mathrm{DOM}}-Q_{w, \mathrm{RT}}}{Q_{w, \mathrm{RT}}}\right| \times 100$

with RT standing for the ray tracing. $E_{\text {Mean }}$ is simply the averaged values of relative errors calculated at several points. For an intermediate value of $\kappa\left(\kappa=1.0 \mathrm{~m}^{-1}\right)$, the errors are also provided in the two tables.

For the wall heat flux, the solutions computed with the three numerical schemes are in a good agreement. Nevertheless, we can notice that the "Diamond Mean Flux Scheme" gives significantly better results than the exponential scheme for $\kappa=10.0 \mathrm{~m}^{-1}$. The DMFS remains accurate enough for a large range of optical thicknesses. 

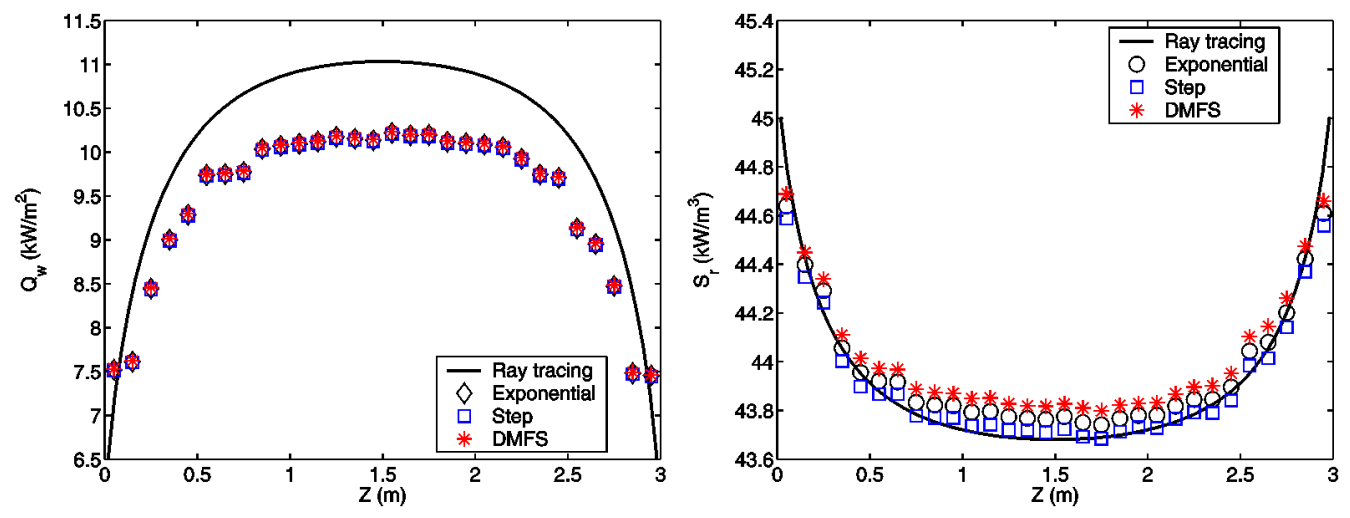

(a)
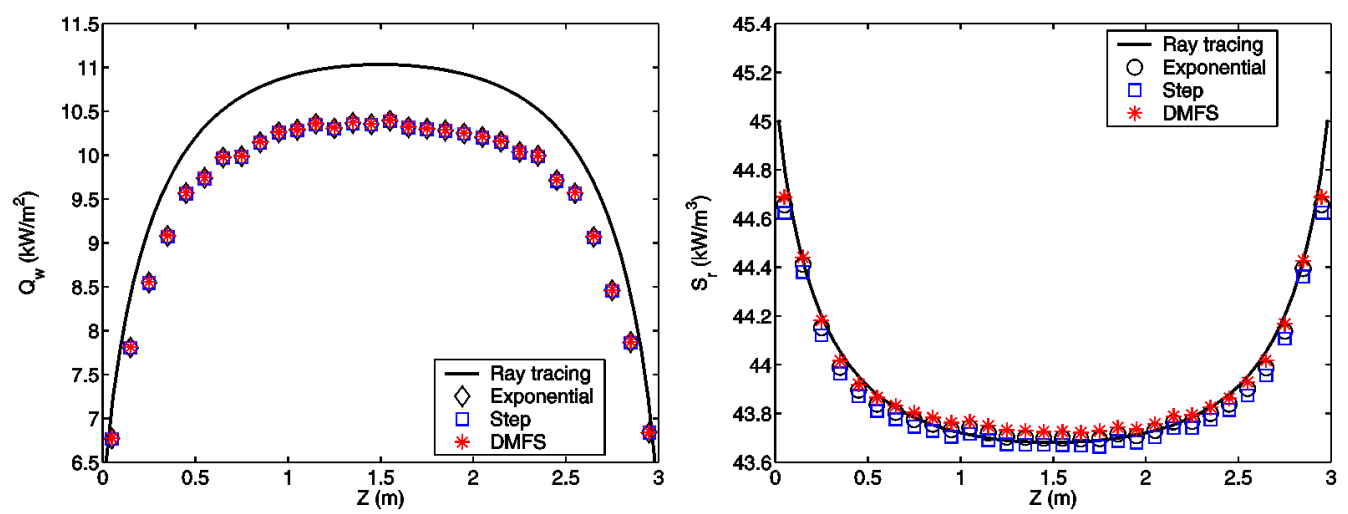

(b)
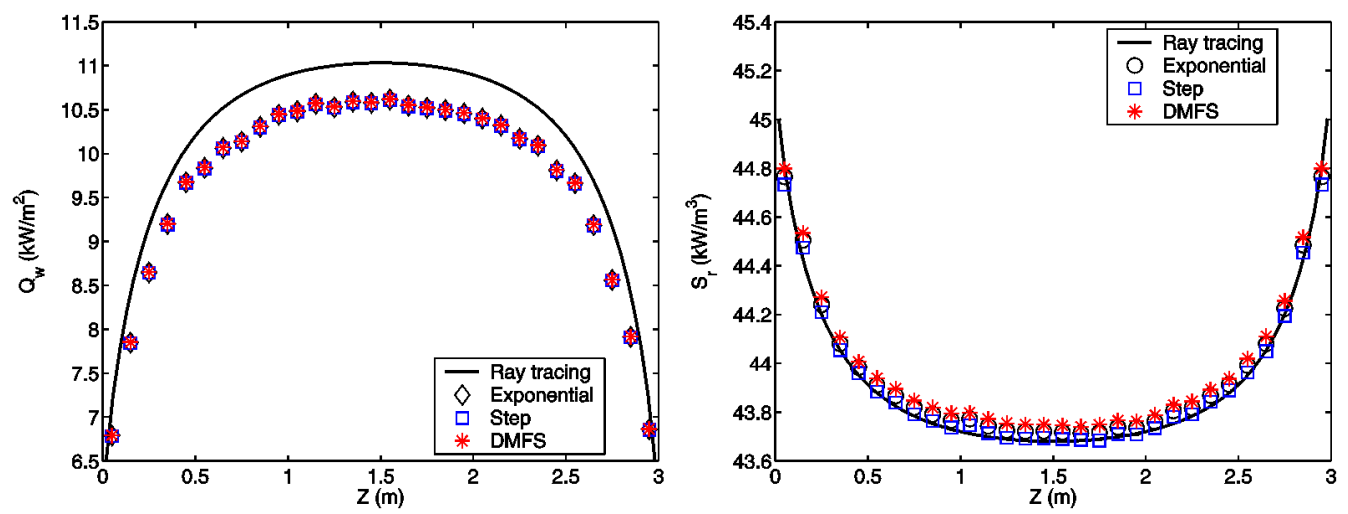

(c)

Fig. 4. $Q_{w}$ on the side wall and $S_{r}$ on the central axis for $\kappa=0.1 \mathrm{~m}^{-1}$ of a cylindrical enclosure with participating gray medium: (a) Unstructured coarse grid (18 920 cells) and angular quadrature $S_{8}$; (b) Unstructured fine grid (140010 cells) and angular quadrature $S_{8}$; (c) Unstructured fine grid (140 010 cells) and angular quadrature $S_{12}$.

\subsection{Accuracy levels for combustion applications: The high absorption limit under the finite volume approximation}

The preceding academic test cases have pointed out two difficulties commonly encountered with such numerical approaches: the ray effect and the convergence difficulties at the optically thick absorption limit. Ray effects are very much dependent on the geometry of the system and can only be reduced by the increase of the number of discrete directions. As far as the optically thick absorption limit is concerned, two remarks can be drawn that motivate further discussions in the present section:

(1) One would expect that the sophisticated exponential scheme lead to better convergence quality than the simple step scheme and the DMFS; which is not the case in the black walled cylindrical enclosures case.

(2) High absorption optical thicknesses are very commonly encountered in combustion at the center of $\mathrm{CO}_{2}$ and $\mathrm{H}_{2} \mathrm{O}$ infrared absorption lines, which raises the question 

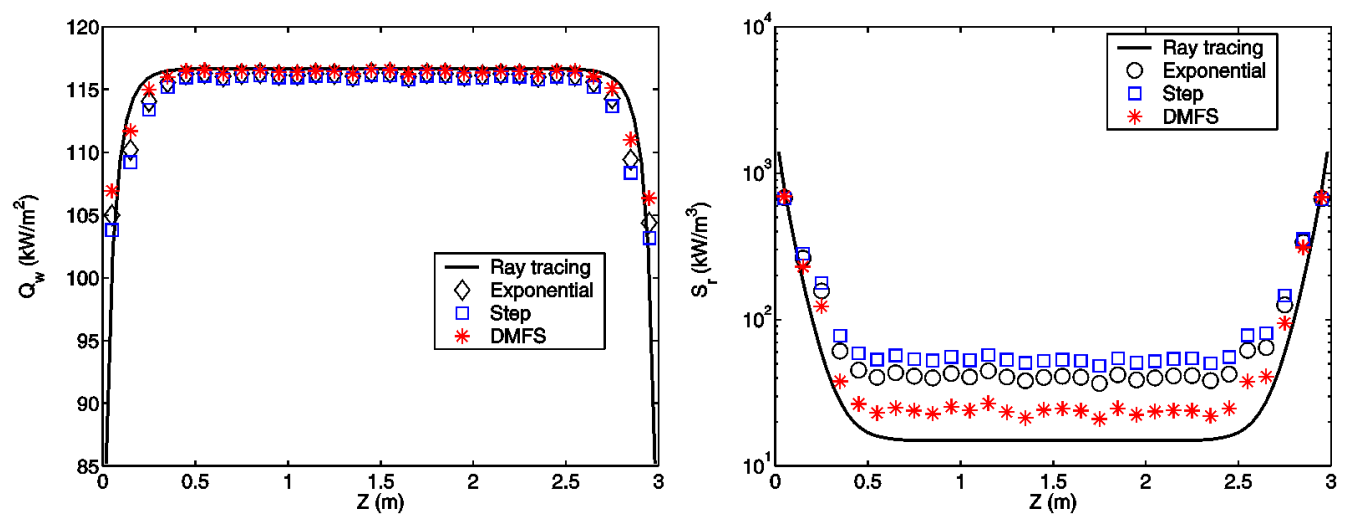

(a)
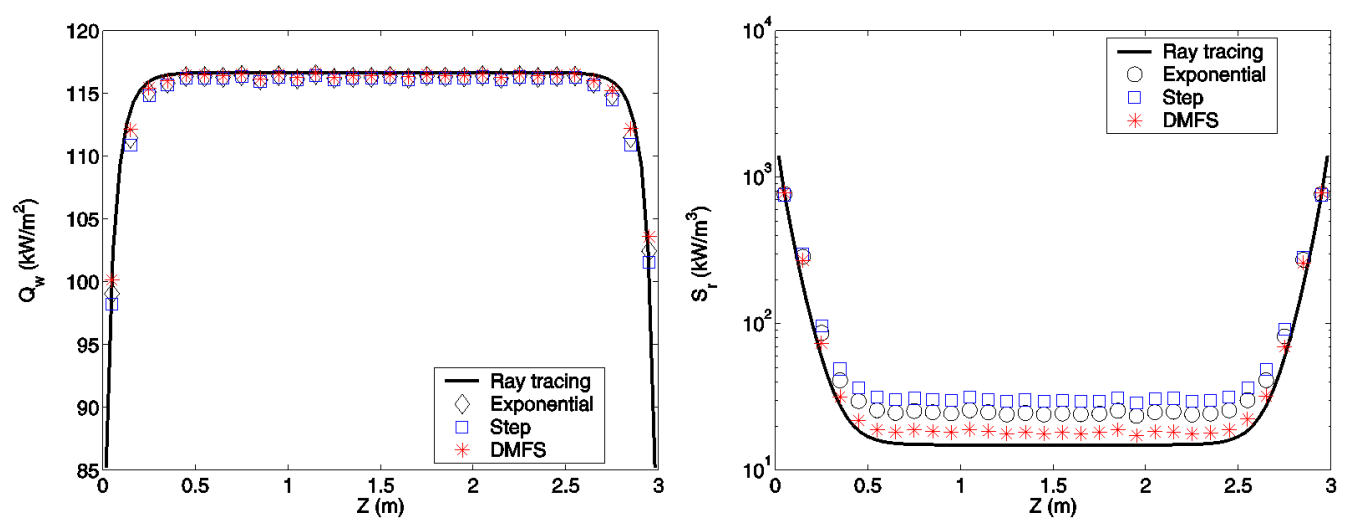

(b)
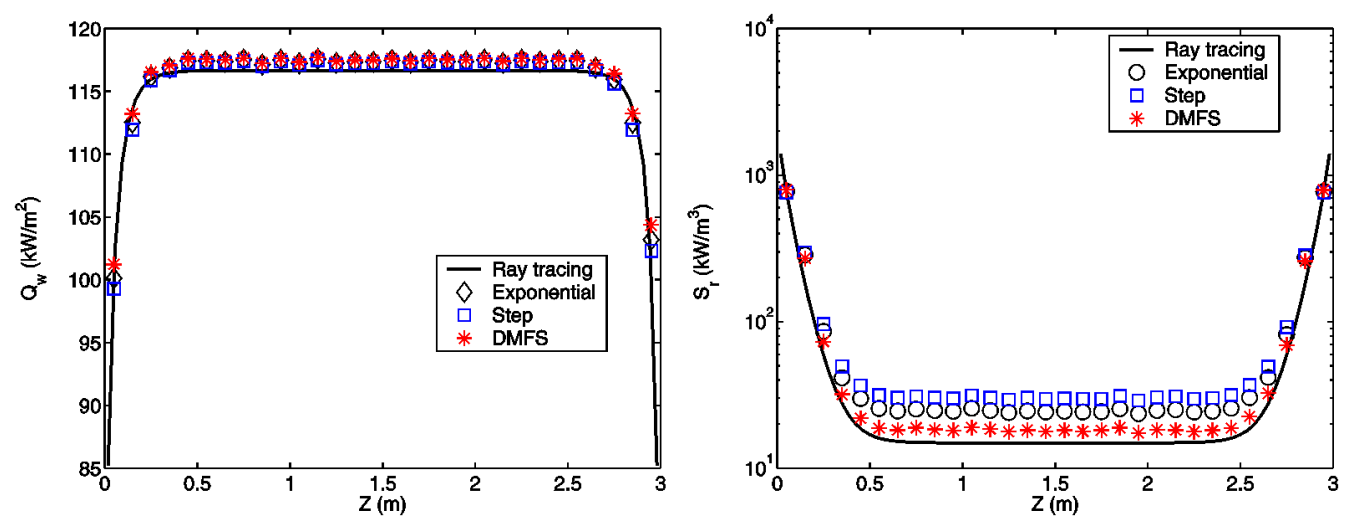

(c)

Fig. 5. $Q_{w}$ on the side wall and $S_{r}$ on the central axis for $\kappa=10.0$ : (a) Unstructured coarse grid (18920 cells) and angular quadrature $S_{8}$; (b) Unstructured fine grid (140010 cells) and angular quadrature $S_{8}$; (c) Unstructured fine grid (140010 cells) and angular quadrature $S_{12}$.

of the general meaning of DOM-FVM computations for combustion applications.

In the present section, we argue that the convergence difficulties at the high absorption limit are intrinsically associated with the finite volume approximation and that, for reasons related to spectral correlation effects, accurate radiative heat source fields can be produced with such methods for combustion applications despite of extreme absorption encountered in $\mathrm{CO}_{2}$ and $\mathrm{H}_{2} \mathrm{O}$ lines centers, in meter scale configurations.

\subsubsection{Black walled spherical enclosure: A parametric} study of the optical thickness influence

To analyze in more details the trends identified here above, this new section presents a simple test case which provides an analytical solution (of the source term only) in order to further understand, via a parametric study, the influence of the optical thickness on the source term calculations. In this test case, a sphere with a radius $R=1 \mathrm{~m}$ has been considered and two different grids have been used (Fig. 6): a coarse one (18920 cells) and very fine one (140010 cells). 
In a first case, the medium is isothermal at $T_{\max }=1200 \mathrm{~K}$ and the wall is black at $T_{\min }=300 \mathrm{~K}$. In a second test case, wall conditions are identical but the temperature of the medium is depending on the space variable $r$ as follows:

$T(r)=\left(T_{\max }^{4}\left(1-r^{2}\right)+T_{\min }^{4} r^{2}\right)^{1 / 4}$

The source term $S_{r, C}$ is evaluated at the center of the sphere using a $S_{12}$ quadrature with the three different spatial differ-

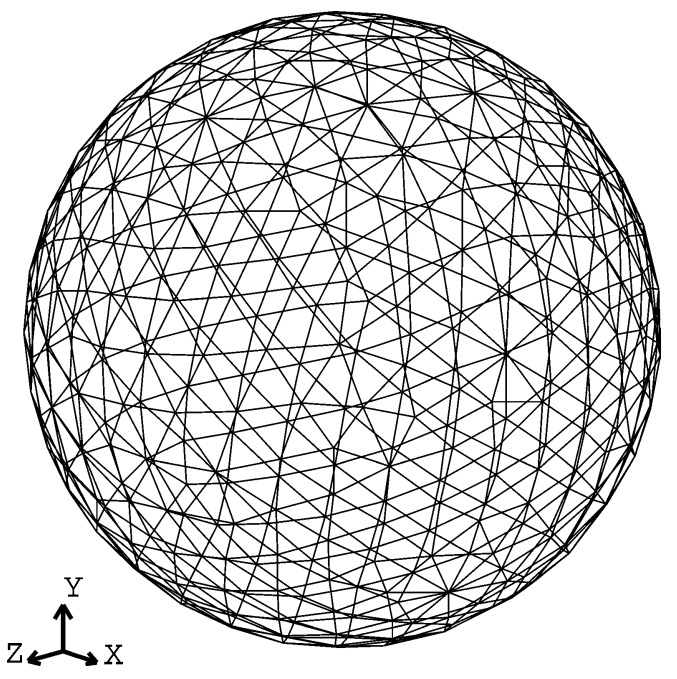

Fig. 6. Spherical enclosure grid. encing schemes studied in the previous section. Results are compared to the analytical solution obtained considering the spherical symmetry:

$S_{r, C, \text { analytical }}=4 \pi \kappa\left[I_{b}-\left(I_{b, w} e^{-\kappa R}+\kappa \int_{0}^{R} I_{b} e^{-\kappa r} \mathrm{~d} r\right)\right]$

The relative error of the radiative source term is represented versus $\kappa$ in Fig. 7 and is computed as follows:

$E=\left|\frac{S_{r, C, \text { DOM }}-S_{r, C, \text { analytical }}}{S_{r, C, \text { analytical }}}\right| \times 100$

From Fig. 7, we can notice that the relative error increases when the absorption coefficient increases. The change of direction of the curve, in Fig. 7(b) and (d), is only due to the use of the absolute value in the relative error formulation (see Eq. (29)). In the isothermal test case, extremely poor levels of accuracy are observed when increasing optical thickness (Fig. 7(a) and (c)). That phenomenon could be interpreted as the "false scattering" effect (numerical diffusion linked to cell optical thickness) with the use of the finite volume approximation. This observation can be related to the fact that, in numerical experiments, the geometrical mesh structure is kept constant when increasing the absorption coefficient, leading to a regular increase of the optical thickness of each mesh. For the non-isothermal case, the same phenomenon

Table 1

Relative Errors on the radiative source term $S_{r}$

\begin{tabular}{|c|c|c|c|c|c|c|c|c|c|c|}
\hline \multirow{2}{*}{$\begin{array}{l}\text { Absorption coefficient } \\
\text { Relative errors }\end{array}$} & & \multicolumn{3}{|c|}{$\kappa=0.1 \mathrm{~m}^{-1}$} & \multicolumn{3}{|c|}{$\kappa=1.0 \mathrm{~m}^{-1}$} & \multicolumn{3}{|c|}{$\kappa=10.0 \mathrm{~m}^{-1}$} \\
\hline & & $E_{\operatorname{Max}}$ & $E_{\text {Mean }}$ & $E_{\text {Min }}$ & $E_{\text {Max }}$ & $E_{\text {Mean }}$ & $E_{\text {Min }}$ & $E_{\text {Max }}$ & $E_{\text {Mean }}$ & $E_{\text {Min }}$ \\
\hline \multirow[t]{2}{*}{ Coarse mesh and $S_{8}$} & Step scheme & $0.56 \%$ & $0.096 \%$ & $0.0024 \%$ & $3.78 \%$ & $2.60 \%$ & $0.51 \%$ & $328 \%$ & $219 \%$ & $7.67 \%$ \\
\hline & Diam. scheme & $0.35 \%$ & $0.26 \%$ & $0.045 \%$ & $5.12 \%$ & $3.55 \%$ & $0.029 \%$ & $112 \%$ & $56.4 \%$ & $2.83 \%$ \\
\hline \multirow[t]{2}{*}{ Fine mesh and $S_{8}$} & Step scheme & $0.42 \%$ & $0.11 \%$ & $0.0095 \%$ & $2.14 \%$ & $0.95 \%$ & $0.016 \%$ & $111 \%$ & $88.4 \%$ & $6.64 \%$ \\
\hline & Expon. scheme & $0.35 \%$ & $0.077 \%$ & $0.0004 \%$ & $1.89 \%$ & $1.18 \%$ & $0.076 \%$ & $72.2 \%$ & $57.3 \%$ & $5.2 \%$ \\
\hline \multirow[t]{3}{*}{ Fine mesh and $S_{12}$} & Step scheme & $0.17 \%$ & $0.037 \%$ & $0.0006 \%$ & $1.83 \%$ & $1.15 \%$ & $0.14 \%$ & $111 \%$ & $88.3 \%$ & $5.52 \%$ \\
\hline & Expon. scheme & $0.17 \%$ & $0.092 \%$ & $0.033 \%$ & $2.26 \%$ & $1.41 \%$ & $0.65 \%$ & $71.9 \%$ & $57.2 \%$ & $4.07 \%$ \\
\hline & Diam. scheme & $0.24 \%$ & $0.15 \%$ & $0.03 \%$ & $2.71 \%$ & $1.67 \%$ & $0.33 \%$ & $43.6 \%$ & $21.2 \%$ & $1.99 \%$ \\
\hline
\end{tabular}

Table 2

Relative Errors on the radiative heat flux at the wall $Q_{w}$

\begin{tabular}{|c|c|c|c|c|c|c|c|c|c|c|}
\hline \multirow{2}{*}{$\begin{array}{l}\text { Absorption coefficient } \\
\text { Relative errors }\end{array}$} & & \multicolumn{3}{|c|}{$\kappa=0.1 \mathrm{~m}^{-1}$} & \multicolumn{3}{|c|}{$\kappa=1.0 \mathrm{~m}^{-1}$} & \multicolumn{3}{|c|}{$\kappa=10.0 \mathrm{~m}^{-1}$} \\
\hline & & $E_{\text {Max }}$ & $E_{\text {Mean }}$ & $E_{\text {Min }}$ & $E_{\text {Max }}$ & $E_{\text {Mean }}$ & $E_{\text {Min }}$ & $E_{\text {Max }}$ & $E_{\text {Mean }}$ & $E_{\text {Min }}$ \\
\hline \multirow[t]{2}{*}{ Coarse mesh and $S_{8}$} & Step scheme & $11.0 \%$ & $7.39 \%$ & $4.46 \%$ & $8.91 \%$ & $3.79 \%$ & $0.40 \%$ & $4.55 \%$ & $0.78 \%$ & $0.41 \%$ \\
\hline & Diam. scheme & $10.8 \%$ & $7.18 \%$ & $4.72 \%$ & $10.7 \%$ & $2.45 \%$ & $0.12 \%$ & $7.15 \%$ & $0.61 \%$ & $0.077 \%$ \\
\hline \multirow[t]{3}{*}{ Fine mesh and $S_{8}$} & Step scheme & $7.78 \%$ & $5.92 \%$ & $2.64 \%$ & $3.72 \%$ & $1.94 \%$ & $0.55 \%$ & $2.33 \%$ & $0.61 \%$ & $0.23 \%$ \\
\hline & Expon. scheme & $7.73 \%$ & $5.86 \%$ & $2.58 \%$ & $3.31 \%$ & $1.60 \%$ & $0.68 \%$ & $2.66 \%$ & $0.44 \%$ & $0.15 \%$ \\
\hline & Diam. scheme & $7.67 \%$ & $5.80 \%$ & $2.51 \%$ & $2.99 \%$ & $1.25 \%$ & $0.26 \%$ & $3.80 \%$ & $0.28 \%$ & $0.058 \%$ \\
\hline \multirow[t]{3}{*}{ Fine mesh and $S_{12}$} & Step scheme & $6.69 \%$ & $4.57 \%$ & $2.54 \%$ & $2.90 \%$ & $1.17 \%$ & $0.62 \%$ & $2.50 \%$ & $0.50 \%$ & $0.054 \%$ \\
\hline & Expon. scheme & $6.63 \%$ & $4.51 \%$ & $2.48 \%$ & $2.49 \%$ & $0.83 \%$ & $0.23 \%$ & $3.40 \%$ & $0.56 \%$ & $0.0037 \%$ \\
\hline & Diam. scheme & $6.57 \%$ & $4.45 \%$ & $2.42 \%$ & $2.88 \%$ & $0.50 \%$ & $0.0006 \%$ & $4.58 \%$ & $0.73 \%$ & $0.27 \%$ \\
\hline
\end{tabular}




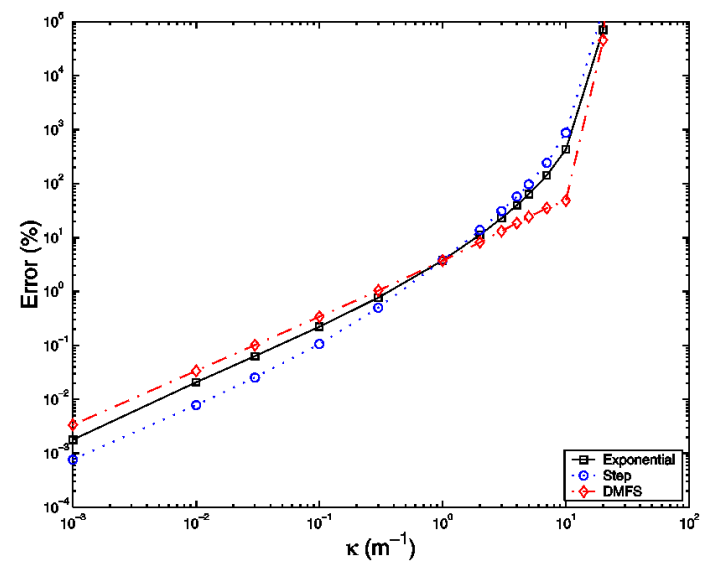

(a)

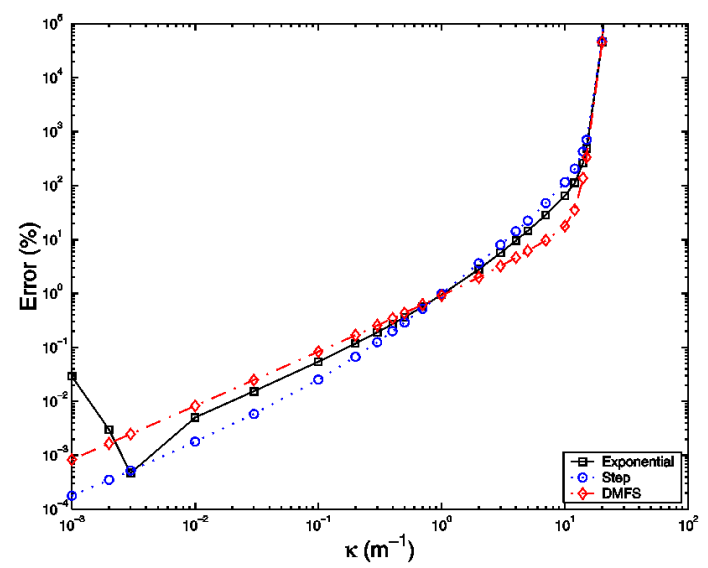

(c)

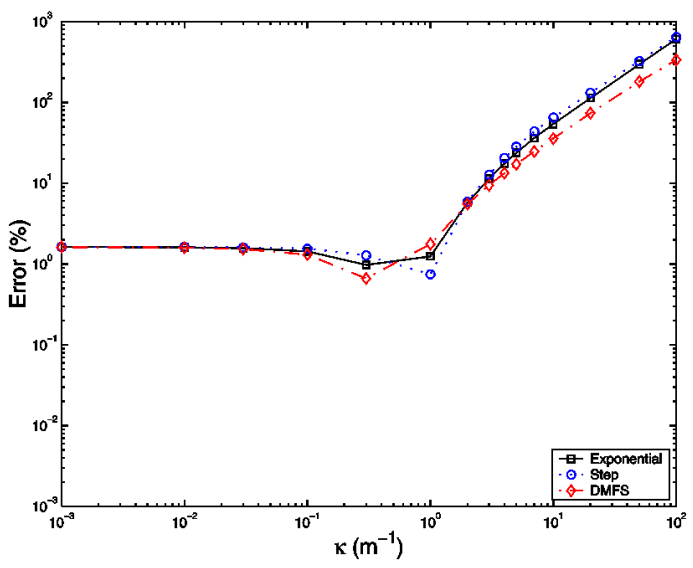

(b)

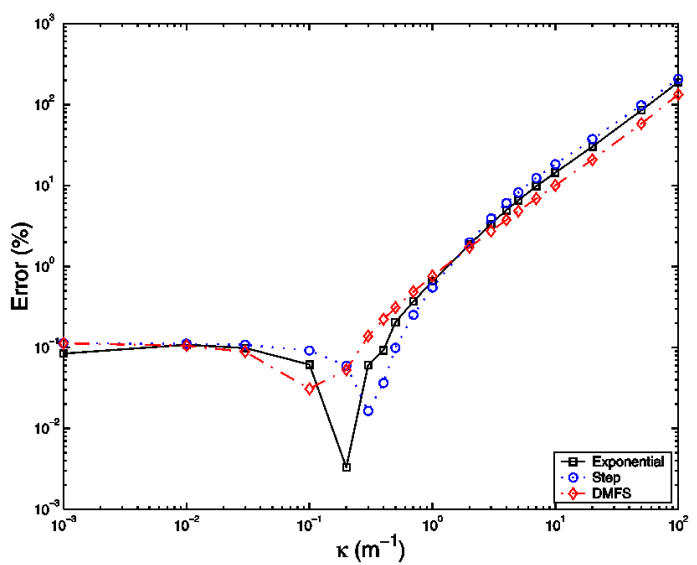

(d)

Fig. 7. Relative Error for $S_{r, C}$ at the center of the sphere: (a) For the isothermal gray medium case using the coarse grid; (b) For the non-isothermal gray medium case using the coarse grid; (c) For the isothermal gray medium case using the fine grid; (d) For the non-isothermal gray medium case using the fine grid.

occurs but radiative exchanges at small distances (exchanges between closest gas volumes) contribute more than the exchanges with the walls. This reduces the optical thicknesses effectively linked to the most contributing exchanges. Consequently, false scattering has less influence and the relative error on the radiative source term is smaller than the one obtained in the isothermal test case. These errors remain however very high when optical thickness reaches values of the order of ten or above, which is commonly encountered at the center of gaseous absorption lines such as those of $\mathrm{H}_{2} \mathrm{O}$ and $\mathrm{CO}_{2}$ in combustion.

\subsubsection{Finite volume approaches in the optically thick absorption limit}

The preceding parametric study has indicated that strong errors are to be expected when increasing the cell absorption optical thickness, whatever the numerical scheme among the three considered ones. This seems in contradiction with the fact that the exponential scheme solves the RTE exactly within each tetrahedrical cell in 3D, or triangular cell in 2D. However, when analyzing this point in detail, it appears that the error is entirely associated with the standard finite vol-

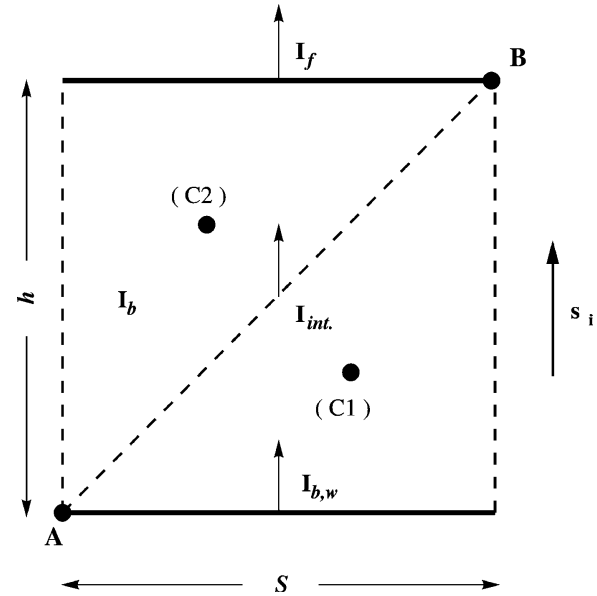

Fig. 8. Simplified configuration.

ume approximation that the intensity is uniform across an upstream face.

This may be seen in the following simple bidimensional case (Fig. 8): an isothermal gray medium of thickness $h$ and absorption coefficient $\kappa$ (the blackbody intensity being $I_{b}$ ), 
between two parallel isothermal black walls of surface $S$ (the blackbody intensity being $I_{b, w}$ ). Let us assume that we want to estimate the integrated intensity $I_{f \text {,total }}$ impinging at the surface in a direction $\vec{s}_{i}$ orthogonal to the two parallel surfaces. The analytical solution is:

$I_{f, \text { total }}=\int_{S} I_{f} \mathrm{~d} S=I_{f} . S$

with

$I_{f}=I_{b, w} e^{-\kappa h}+\kappa \int_{0}^{h} I_{b} e^{-\kappa r} \mathrm{~d} r=I_{b, w} e^{-\tau}+I_{b}\left(1-e^{-\tau}\right)$

where $\tau=\kappa h$ is the absorption optical thickness. Whatever the considered numerical scheme, this geometry requires that the field is divided in at least two triangular cells $(\mathrm{C} 1)$ and (C2), as indicated in Fig. 8. The numerical resolution will therefore include the computation of the averaged intensity $I_{\text {int }}$ at the interface between $(\mathrm{C} 1)$ and (C2).

The analytical expression of $I_{\text {int }}$ can be easily shown to be:

$I_{\text {int }}=I_{b, w} \chi+I_{b}(1-\chi)$

where $\chi$ for a two-dimensional system becomes [12]:

$\chi=\left(1-e^{-\tau}\right) / \tau$

Note that those expressions corresponds to Eqs. (13) and (14) which indicates that the exponential scheme computes $I_{\text {int }}$ exactly from the knowledge of a uniform intensity $I_{b, w}$ at the upstream face of $(\mathrm{C} 1)$, and a uniform emission within the cell. And indeed, the upstream intensity is uniform (emission by the isothermal black surface) and the volume emission is uniform (isothermal medium). At this stage, everything remains therefore exact with a sophisticated enough numerical scheme.

However, things are very different if we now consider (C2) to compute the averaged downstream intensity $I_{f}$ from the knowledge of the averaged upstream intensity $I_{\text {int }}$. As no further information is available, the common finite volume approximation consists in assuming that the intensity is distributed uniformly along the upstream face. Under this assumption, the analytical expression of $I_{f}$ may be shown to be (again, this is what the exponential scheme gives for a uniform intensity at the upstream face as it is exact within each cell):

$I_{f}=I_{\text {int }} \chi+I_{b}(1-\chi)=I_{b, w} \chi^{2}+I_{b}\left(1-\chi^{2}\right)$

This expression is approximate. It only matches Eq. (31) at the optically thin limit. Therefore whatever the quality of the spatial integration scheme, an error will occur at the optically thick limit that is due to the lack of information concerning the distribution of the intensity along the upstream face. For illustration, at the two points A and B reported on the Fig. 8, the intensity is arbitrarily set to $I_{\text {int }}=I_{b, w} \chi+I_{b}(1-\chi)$ whereas its exact value is respectively $I_{\mathrm{A}}=I_{b, w}$ and $I_{\mathrm{B}}=$
$I_{b, w} e^{-\tau}+I_{b}\left(1-e^{-\tau}\right)$. The resulting solutions and associated errors are represented in Fig. 9 as function of the absorption optical thickness $\tau$ for two test cases:

Case 1 (transmission): $I_{b}=0$ et $I_{b, w}=1$;

Case 2 (emission/self-absorption): $I_{b}=1$ et $I_{b, w}=0$.

What these figures tell us when looking at the curves labeled "exponential" is the accuracy loss due to the finite volume approximation itself. In the present configuration this is exactly what the exponential scheme would give because the exponential scheme is exact within each cell. We have also reported in the same figures solution and relative errors (as in Eq. (29)) resulting of the finite volume approximation combined with the simple approximate step and diamond mean flux schemes.

The first comment is that the convergence difficulties at high optical thicknesses are mainly due to the finite volume approximation itself and not to the quality of the numerical schemes. Concerning, the emission and self-absorption modelling, the finite volume approximation is justified when the medium becomes very thick and the error on $I_{f}$ decreases (Fig. 9(b) and (d)). However at such optical thicknesses, the DMFS gives unrealistic negative values for $I_{\text {int }}$ which leads to an increasing error on the final intensity and none of the three numerical schemes succeed in modelling the transmission (very small values for $I_{f}$ ).

As a conclusion, we can notice from Fig. 9(a) and (c) that:

- as long as the DMFS does not calculate negative intensities (for $I_{\text {int }}$ ), it gives the most accurate approximate solution,

- for intermediate optical thicknesses $\left(10^{-1}-10^{+1}\right)$, the finite volume approximation introduces important inaccuracies in the different numerical schemes (see Fig. 9(a) and (c)).

\subsubsection{Accuracy levels associated to finite volume approximations for line spectra of combustion gases}

We have seen that even with high accuracy or exact spatial integration schemes, the usual finite volume approximation itself introduces high uncertainties at the limit of optically thick absorption. A last test case is considered here, which is close to combustion applications, in order to show how the problems encountered at high optical thicknesses (at the centers of gaseous spectral lines) may affect the accuracy of the radiative source term in a real combustion gas. The same unity sphere as above is meshed with 30000 tetrahedra and filled with an isothermal gas containing water vapor and nitrogen. The spectral dependency of the absorption coefficient is here represented with the use of a $S N B$ - $c k$ model [23-25]. The $S N B$ data have been provided by the EM2C laboratory [26]. The source term $S_{r}$ is obtained by computing $N_{\text {bands }} \times N_{\text {quad }}$ gray calculations where $N_{\text {bands }}=367$ is the number of narrow bands which have the same spectral width, and for each narrow band $N_{\text {quad }}=5$ is the number of 


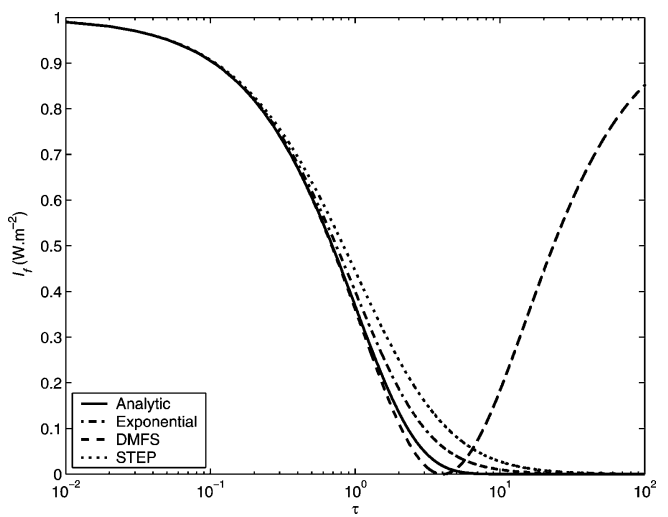

(a)

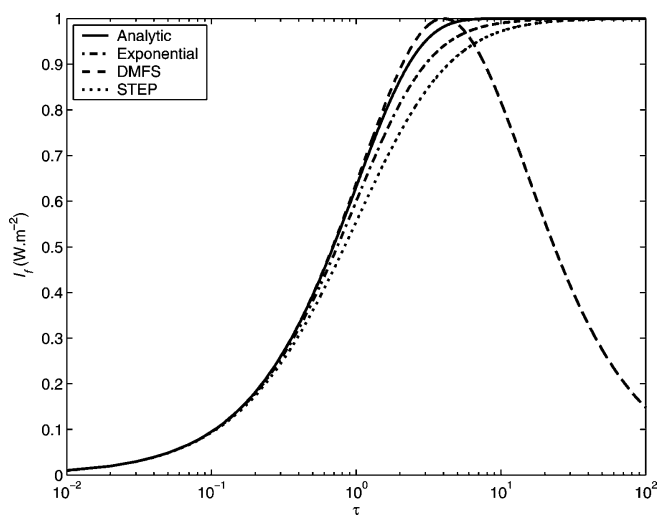

(c)

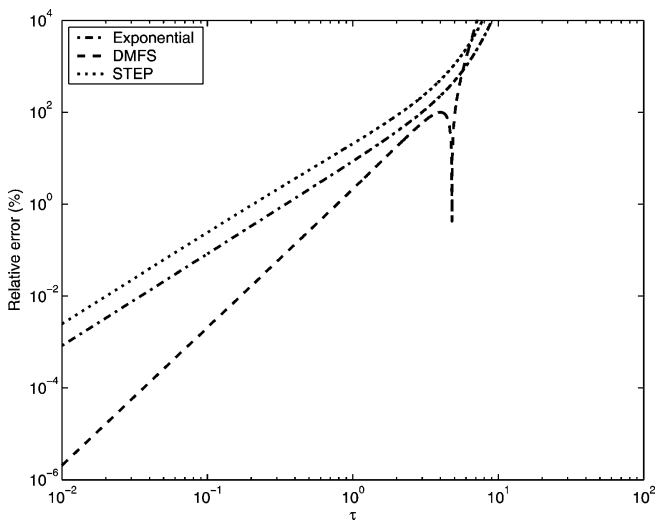

(b)

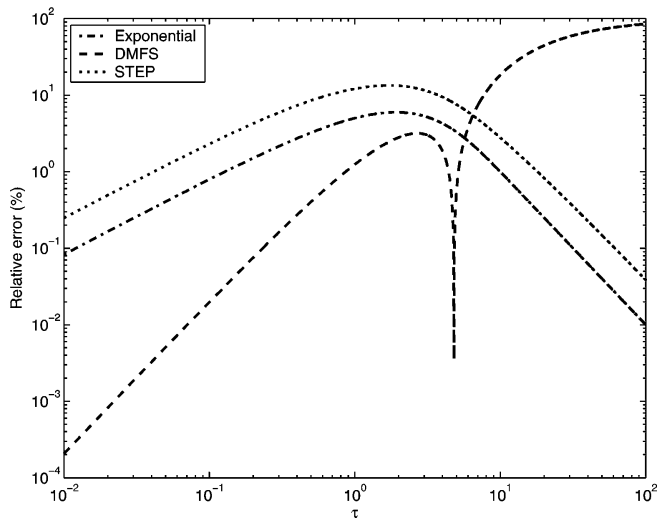

(d)

Fig. 9. Comparison between the four expressions of $I_{f}(k)$ and the relative error for the three differencing schemes: (a) Transmission modelling: $I_{f}(k)$; (b) Transmission modelling: Relative error; (c) Emission/self-absorption modelling: $I_{f}(k)$; (d) Emission/self-absorption modelling: Relative error.

Gauss-Legendre quadrature points used in the approximation of the spectral integration. Then, the analytical solution integrated over the whole spectrum is written as:

$$
\begin{aligned}
S_{r, C, \text { analytic }}= & \sum_{i=1}^{N_{\text {band }}} \sum_{j=1}^{N_{\text {quad }}} 4 \pi \Delta v_{i} w_{i j} \kappa_{i j} e^{-\kappa_{i j} R} \\
& \times\left(I_{b, C, i j}-I_{b, w, i j}\right)
\end{aligned}
$$

Consequently, using the DOM, the source term [27] is obtained as follows:

$S_{r, \mathrm{DOM}}=\sum_{i=1}^{N_{\text {band }}} \sum_{j=1}^{N_{\text {quad }}} \Delta v_{i} w_{i j} \kappa_{i j}\left(4 \pi \bar{I}_{b, i j}-G_{i j}\right)$

where $G_{i j}$ is obtained from Eq. (7) for $\kappa=\kappa_{i j}$.

To study the effect of the angular quadrature on the estimation of $S_{r, C}$, the relative error has been computed according to Eq. (29) in a first configuration with $X_{\mathrm{H}_{2} \mathrm{O}}=0.2$ and $X_{\mathrm{N}_{2}}=0.8 . S_{4}, S_{8}, S_{12}$ and $L C_{11}$ [22] have been tested using the DMFS scheme and for each quadrature, the relative error is about $1.2 \%$.

Consequently, the $S_{4}$ quadrature is chosen and the relative error of the source term is computed for the three numerical schemes (with the parameter $X_{\mathrm{H}_{2} \mathrm{O}}$ varying). Fig. 10 represents the relative error versus the molar concentration of water vapor. As we noticed previously, the relevant parameter that influences the solution accuracy is the optical thickness which is directly related to the water vapor molar fraction $X_{\mathrm{H}_{2} \mathrm{O}}$. The sensitivity of the solution accuracy to the molar fraction of water vapor is illustrated in Fig. 10(a). For small molar fractions $(X=0.01)$, the exponential scheme could not provide an acceptable physical solution because of the sign of the intensity $I_{p}$ which becomes negative. A very small discrepancy between the three schemes is observed, with a relatively better accuracy of the DMFS. Altogether, the accuracy level is much better than what could be expected on the basis of preceding analysis. As the medium is isothermal, the radiative exchanges with the walls are the only relevant ones. They occur at a long distance, which means that the exchanges corresponding to low absorption coefficients contribute more than the one at high values where extinction rapidly takes place. Finally, the last figure Fig. 10(b) deals with a very thick medium where soot particles are added to water vapor and nitrogen, taking into account a very high soot volumetric fraction $f_{v}=10^{-5}$. The total absorption coefficient is expressed by

$\kappa_{i j}=\kappa_{i j, \text { gas }}+\kappa_{i, \text { soot }}$ 


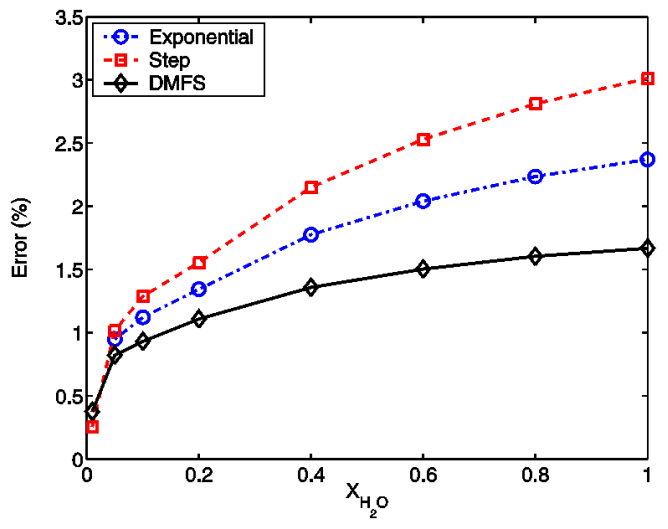

(a)

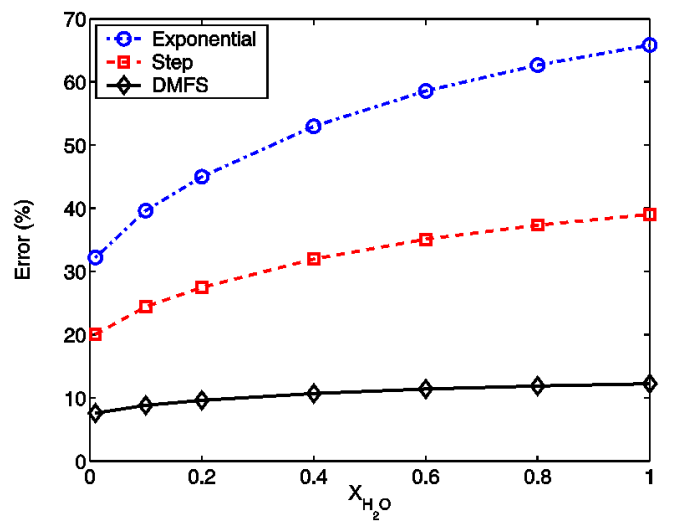

(b)

Fig. 10. Relative Error for $S_{r, C}$ at the center of the sphere using the medium grid: (a) Case of the isothermal gaseous medium; (b) Case of the isothermal mixture of gas and soot.

with [28]:

$\kappa_{i, \text { soot }}=5.5 f_{v} v_{i}$

where $f_{v}$ stands for the soot volumetric fraction and $v_{i}$ is the wave number taken at the center of a narrow band. As soot radiation becomes dominant, the medium tends to become thick and gray. Then, the results obtained in Fig. 10(b) show big discrepancies between the three schemes which confirm the trends of the relative error for $S_{r}$ observed in the first cylinder test case and a good behavior of the DMFS. In this case, because of the gray behavior of soot in each narrow band, each absorption coefficient is increased at the same level and leads to optically thick media. All the frequencies contribute similarly to the estimation of the exchange with the wall, consequently we can use similar explanations than those presented in the gray case.

Altogether, these results only confirm the good behavior of the code giving satisfactory accuracy levels for well mixed combustion chambers, where the gas volume can be considered as quasi-isothermal, with intermediate soot concentration levels. It has been shown in the previous section that, for non-isothermal media, the main exchanges contributing to the radiative source term evaluation are the exchanges with the gas at short or intermediate distance. Therefore, a final interesting test case considering the spherical enclosure containing non-isothermal and non-gray medium is necessary but is not presented here because no analytical solution along the radius is available. A Monte Carlo calculation is presently being performed, that will serve as a reference solution for an extension of the present analysis.

\section{Concluding remarks}

A DOM code using unstructured grids has been developed for coupling with finite volume CFD-combustion code and has been validated using academic test cases. This study emphasizes the influence of the absorption optical thickness on the accuracy level. We could then show that the difficulties encountered at high optical thicknesses were associated with the standard finite volume approximation where the intensity is distributed uniformly along each upstream face. These difficulties are therefore quite independent of the sophistication level of the spatial differencing scheme. In particular, for such applications as combustion ones, where the constraints in term of computational times are strong, it appears that there is no particular needs for a very accurate but complex scheme such as the exponential scheme, considering that a simple scheme such as the DMFS insures comparable accuracy levels up to intermediate optical thicknesses and encounter the same strong difficulties at the high absorption limit.

These strong difficulties could lead to believe that standard finite volume approximations are useless in the combustion context where strong absorption commonly appears at the center of infrared $\mathrm{CO}_{2}$ and $\mathrm{H}_{2} \mathrm{O}$ absorption lines. Parametric test cases were considered to check this point and it appeared that when real gas spectra are considered for integrated radiation computations, much less difficulties are met, in terms of accuracy, than what could be expected on the basis of the conclusions of the gray medium analysis.

This conclusion could only be drawn for well mixed combustion chambers and the cases with strongly heterogeneous temperature fields require further analysis.

For such heterogeneous cases, significant short distance radiative exchanges may appear in optically thick spectral regions. As soon as such exchanges play a significant role the standard finite volume approximation will lead to strong errors. It will therefore be required that some informations are kept concerning the distribution of the intensity along the upstream faces. These ideas have been explored by several authors with the finite element approach where the intensity is stored at each nodes (at the cell vertices), which is the better start basis for the description of its distribution along the face [29-31]. These techniques have been shown to be very efficient at the limit of strong optical thicknesses. It would be worth looking at their behavior in the limit of high absorp- 
tion optical thicknesses for combustion applications. However, in the short term, considering today's computational constraints in this field, such approaches could only be considered for computational accurate reference solutions but not for coupling with finite volume CFD-combustion codes. In the long term, it would certainly be of great interest to try and combine Sakami's exponential scheme with such finite element approaches.

\section{Acknowledgements}

The financial support of the European Commission in the framework of the TMR programme (RADIARE project, No. ERBFMRX-CT98-224) is gratefully acknowledged. Special thanks for the Pr. Coelho of the IST in Lisboa for his indications and fruitful discussions.

\section{References}

[1] S. Chandrasekhar, Radiative Transfer, Clarendon Press, Oxford, 1950.

[2] B.G. Carlson, K.D. Lathrop, Transport theory - The method of discrete ordinates, in: Computing in Reactor Physics, Gordon\&Breach, 1968.

[3] W.A. Fiveland, Three-dimensional radiative heat transfer solutions by the discrete ordinates method, J. Thermophys. 2 (4) (1988) 309-316.

[4] W.A. Fiveland, A.S. Jamaluddin, Three-dimensional spectral radiative heat transfer solutions by the discrete ordinates method, J. Thermophys. 5 (3) (1991) 335-339.

[5] W.A. Fiveland, J.P. Jessee, Comparison of discrete ordinates method formulations for radiative heat transfer in multidimensional geometries, J. Thermophys. Heat Transfer 9 (1) (1995) 47-54.

[6] J.S. Truelove, Three-dimensional radiation in absorbing-emittingscattering in using the discrete ordinates approximation, J. Quant. Spectrosc. Radiat. Transfer 39 (1988) 27-31.

[7] A.S. Jamaluddin, P.J. Smith, Discrete ordinates solution of radiation transfer equation in nonaxisymmetric cylindrical enclosures, J. Thermophys. Heat Transfer 6 (2) (1992) 242-245.

[8] G.D. Raithby, E.H. Chui, A finite volume method for predicting a radiant heat transfer in enclosures with participating media, J. Heat Transfer 12 (1990) 415-423.

[9] J.C. Chai, G. Parthasarathy, H.S. Lee, S.V. Patankar, Finite volume radiative heat transfer procedure for irregular geometries, J. Thermophys. Heat Transfer 9 (3) (1995) 410-415.

[10] J.Y. Murthy, S.R. Mathur, Finite volume method for radiative heat transfer using unstructured meshes, J. Thermophys. Heat Transfer 12 (3) (1998) 313-321.

[11] J.P. Moder, G.N. Kumar, J.C. Chai, An unstructured-grid radiative heat transfer module for the national combustion code, American Institute of Aeronautics and Astronautics, AIAA-2000-0453, 2000.

[12] M. Sakami, A. Charette, V. Le Dez, Application of the Discrete Ordinates Method to combined conductive and radiative heat transfer in a two-dimensional complex geometry, J. Quant. Spectrosc. Radiat. Transfer 56 (4) (1996) 517-533.

[13] M. Sakami, A. Charette, V. Le Dez, Radiative heat transfer in 3dimensional enclosures of complex geometry by using the discrete ordinates method, J. Quant. Spectrosc. Radiat. Transfer 59 (1/2) (1998) $117-136$.
[14] M. Sakami, A. Charette, A new differencing scheme for the Discrete Ordinates Method in complex geometries, Rev. Gén. Thermique 37 (1998) 440-449.

[15] J. Liu, H.M. Shang, Y.S. Chen, T.S. Wang, Development of an unstructured radiation model applicable for two dimensional planar, axisymmetric and 3-dimensional geometries, J. Quant. Spectrosc. Radiat. Transfer 66 (2000) 17-33.

[16] J.C. Chai, S.V. Patankar, H.S. Lee, Evaluation of spatial differencing practices for the discrete ordinates method, J. Thermophys. Heat Transfer 8 (1) (1994) 140-144.

[17] J. Ströhle, U. Schnell, K.R.G. Hein, A mean flux discrete ordinates interpolation scheme for general coordinates, in: 3rd International Conference on Heat Transfer, Antalya, 2001.

[18] S.H. Seo, T.K. Kim, Study on interpolation schemes of the discrete ordinates interpolation method for three-dimensional radiative transfer with nonorthogonal grids, ASME J. Heat Transfer 120 (1998) 10911094.

[19] H. Cha, T.H. Song, Discrete ordinates interpolation method applied to irregular three-dimensional geometries, ASME J. Heat Transfer 122 (2000) 823-827.

[20] H.M. Koo, R. Vaillon, V. Goutière, V. Le Dez, H. Cha, T.H. Song, Comparison of three discrete ordinates methods applied to twodimensional curved geometries, Internat. J. Thermal Sci. 42 (2003) 343-359.

[21] R. Vaillon, M. Lallemand, D. Lemonnier, Radiative heat transfer in orthogonal curvilinear coordinates using the discrete ordinates method, J. Quant. Spectrosc. Radiat. Transfer 55 (1996) 7-17.

[22] R. Koch, R. Becker, Evaluation of the quadrature schemes for the discrete ordinates method, in: P. Lybaert, V. Feldheim, D. Lemonnier, N. Selçuk (Eds.), Proceedings of Eurotherm73 on Computational Thermal Radiation in Participating Media, in: Eurotherm Series, vol. 11, Elsevier, Paris, 2003, pp. 59-74.

[23] F. Liu, G.J. Smallwood, Ö.L. Gülder, Band lumping strategy for radiation heat transfer calculations using a Narrowband model, J. Thermophys. 14 (2) (2000) 278-281.

[24] F. Liu, G.J. Smallwood, Ö.L. Gülder, Application of the statistical narrow-band correlated-k method to low-resolution spectral intensity and radiative heat transfer calculations-Effects of the quadrature scheme, Internat. J. Heat Mass Transfer 43 (2000) 3119-3135.

[25] F. Liu, G.J. Smallwood, Ö.L. Gülder, Application of the statistical narrow-band correlated-k method to non-grey gas radiation in $\mathrm{CO}_{2}-\mathrm{H}_{2} \mathrm{O}$ mixtures: Approximate treatments of overlapping bands, J. Quant. Spectrosc. Radiat. Transfer 68 (2001) 401-417.

[26] A. Soufiani, J. Taine, High temperature gas radiative property parameters of statistical narrow band model for $\mathrm{H}_{2} \mathrm{O}, \mathrm{CO}_{2}$ and $\mathrm{CO}$ and correlated-K model for $\mathrm{H}_{2} \mathrm{O}$ and $\mathrm{CO}_{2}$, Internat. J. Heat Mass Transfer 40 (4) (1997) 987-991.

[27] P.J. Coelho, P. Perez, M. El Hafi, Benchmark numerical solutions for radiative heat transfer in two-dimensional nongray sooting media, $\mathrm{Nu}$ mer. Heat Transfer B Fund. 43 (2003) 425-444.

[28] S.C. Lee, C.L. Tien, Optical constants of soot in hydrocarbon flames, in: Eighteenth Symposium (International) on Combustion, 1981, pp. 1159-1166.

[29] M.L. Adams, Subcell balance methods for radiative transfer on arbitrary grids, Transport Theory Statist. Phys. 26 (4\&5) (1997) 385-431.

[30] T.A. Wareing, J.M. McGhee, J.E. Morel, S.D. Pautz, Discontinuous finite element $S_{N}$ methods on three-dimensional unstructured grids, Nuclear Sci. Engrg. 138 (2001) 256-268.

[31] A. Dedner, P. Vollmöller, An adaptative higher order method for solving the radiation transport equation on unstructured grids, J. Comput. Phys. 178 (2002) 263-289. 